\title{
Ein anderer Weg bei dem Logarithmusunterricht: Ein entwickelndes Unterrichtsexperiment
}

\author{
FERENC VÁRADY
}

\begin{abstract}
In my developmental experiment I tried to fusion the expectations of the Hungarian education and the realistic mathematics education. The duration of this experiment was 33 lectures long. In this article I try to show how were introduced the definition, the rules of logarithm with real life problems and the outcome of the experiment.
\end{abstract}

Key words and phrases: definition of logarithm, logarithmical rules, realistic mathematics.

ZDM Subject Classification: C70, D40, D60, D80.

\section{Einführung}

In den vergangenen fünfzehn Jahren, seitdem ich Mathematik in verschiedenen Mittelschulen unterrichte, habe ich gemerkt, dass die Bildungspolitik und die Didaktiker im Unterricht immer wieder neue Wege suchen und einführen. Es reicht, wenn man an den Unterschied zwischen dem "alten" und "neuen" Abitur denkt. Dann kann man leicht verstehen wie sich die Erwartungen im Mathematikunterricht gegenüber Schülern und Lehrern verändert haben. Die Aufgabe der Schüler und des Lehrers war in dieser Situation durch die Zweisprachigkeit erschwert. Kein Schüler war von der Muttersprache her Deutscher, auch meine Muttersprache ist ungarisch. Ich versuchte während des Unterrichts immer neue Aufgabenstellungen, neue Methoden, verschiedene Sozialformen auszuprobieren. Auch kleine Spiele, die Schüler nicht ausschließlich durch die Noten, durch Druck 
anregen können. Auf diesem Weg kam ich zu der Freudenthal'schen Schule, und anhand der didaktischen Grundlagen ihres Mathematikunterrichts habe ich einen Unterrichtsplan für 33 Unterrichtsstunden mit den dazugehörenden Unterrichtsmaterialien zusammengestellt. In diesem Artikel will ich die Methode zeigen, wie der Begriff des Logarithmus und die Logarithmusgesetze eingeführt wurden, wie die Ergebnisse und die Reaktionen der Schüler waren.

\section{Theoretischer Hintergrund}

\subsection{Freudenthal'sche Methode - der realistische Mathematikunterricht}

In Europa und in den USA begannen in den sechziger Jahren die reformpädagogischen Bewegungen im Bereich Mathematik. Das Problem, durch abstrakte Definitionen den Lehrstoff einzuführen und so den mathematischen Inhalt den Schülern beizubringen, hat Skemp zutreffend formuliert:

"Anhand einer Definition kann man niemandem einen höheren Begriff beibringen, ausschließlich nur so, dass man eine große Anzahl von entsprechenden Beispielen darstellt." (Skemp [5], S. 42). Ein anderer Gehirnforscher formuliert das Lernprozess, in erster Linie auf dem Gebiet der Mathematik folgendermaßen: "Lernen geschieht primär über das episodisch-kontextuelle Gedächtnis, d.h. über Inhalte, die mit mir und meiner Umgebung zu tun haben. Abstraktes Wissen ist kontextlos und deshalb direkt schwer zu vermitteln. Abstraktes Wissen entsteht normalerweise über eine Filterung episodischen Wissens durch zunehmenden Fortfall des Kontextes. Dies ist der Grund, warum es gut ist, Inhalte "lebensnah" und kontextreich darzubieten. Dies ist allerdings für den Mathematikunterricht ein besonderes Problem, da es einerseits um die Anwendbarkeit, anderseits auch um ein "tieferes" Verständnis" (Roth [4]).

In erster Linie in den Ländern Schweden, Dänemark und Holland bildet die mathematische Modellierung einen Teil des Lehrstoffes, sie ist darin stark integriert. Seit Anfang der 2000er Jahre haben die Ergebnisse der internationalen PISA-Tests $^{1}$ gezeigt, dass in den Ländern, wo das Modellieren keine besondere Rolle spielt, die Schüler mit realistischen Problemen halb so gut umgehen können, wie die Mitschüler in den erwähnten Ländern. Der realistische Mathematikunterricht entstand in den sechziger Jahren, als die Mathematikdidaktiker

${ }^{1}$ Programme for International Student Assessment (Programm zur internationalen Schülerbewertung) ist seit 2000 alle drei Jahren in den OECD Ländern und ihrer Partnerstaaten durchgeführt. 
eine starke Kritik ausübten, die die "Nutzlosigkeit" der mathematischen Kenntnisse ausdrückten. $\mathrm{Zu}$ diesen Didaktikern gehörten unter anderem Freudenthal, Pollak, Krygovska, Engel, Steiner. Das realistische Problem bedeutet nicht unbedingt, dass man Probleme aus dem "leben" nehmen sollte, sondern dass diese Probleme nah den Schülern stehen müssten. Nach der Meinung von De Lange (De Lange (1996) [3]) können diese Situationen auch mathematische Modelle, Modellierung bedeuten. Zweitens müsste der Mathematikunterricht eine Möglichkeit zur (Neu)Entdeckung bieten. Treffers (Treffers (1987) [6]) unterscheidet in dem Vorgang der Mathematisation zwei Typen, die horizontale und die vertikale Mathematisation. In der horizontalen Mathematisation kommt man von der intuitiven, kontextabhängigen, informalen Ebene mit Hilfe von visuellen Modellen, ModellSituationen, verschiedenen Materialien, Schemen, Symbolen und Diagrammen auf die reflektive, formale, systematische Ebene.

In meinem Unterrichtsexperiment kam diese horizontale Mathematisation betont vor. Ich habe bewusst danach gestrebt, dass die Schüler zuerst nicht den abstrakten mathematischen Sachverhalt kennenlernen. Ich wollte erreichen, dass sie sehen, dass es alltägliche Probleme gibt, die mit ihren bisherigen mathematischen Kenntnissen noch nicht lösbar ist, aber sie fähig dazu sind, das neue Problem zu verstehen, sogar mit einer kleinen Hilfe sie lösen zu können. In der vertikalen Mathematisation baut man die Strukturen, die systematischen, formalen Kenntnisse aus. Ich finde es auch sehr wichtig. Aus diesem Grund haben wir zum Teil gemeinsam, zum Teil die Schüler selbständig die Logarithmusgesetze "entdeckt", aber nachher haben wir sie mit exakten mathematischen Bezeichnungen niedergeschrieben und bewiesen. Ich möchte hier betonen, dass meiner Ansicht nach, alleine das Modellieren, die realistische Methode habe ich nicht ausreichend gefunden, die exakten mathematischen Sachverhalten beizubringen. Nach der Einführung im Bereich der exponentiellen und logarithmischen Vorgängen, bzw. nach den "Entdeckungen" der Logarithmusgesetze haben wir auch den mathematischen Hintergrund mit mathematischen Mitteln beschrieben. Obwohl die Mehrheit der Schüler mittelmäßig oder schwach war, gab es in der Gruppe 2-3 gute Schüler, die vorhatten, eventuell an Hochschulen oder Universitäten zu studieren, wo auch Mathematik vorkommt.

Die didaktischen Richtlinien des realistischen Mathematikunterrichts hat Freudenthal formuliert und man kann sie folgendermaßen zusammenfassen: 
- Mathematik im Kontext: Die mathematische Aktivität der Schüler passiert in einem konkreten Kontext. Die Schüler lernen die Theorie, die Zusammenhänge im konkreten Textumfeld kennen. Aus diesen Erkenntnissen abstrahieren sie später die exakten mathematischen Inhalte.

- Horizontale und vertikale Mathematisation (s. oben);

- Die Wichtigkeit der eigenen Produkte, Konstruktionen der Schüler;

- Sozialer Kontext, Interaktionen;

- Zusammenhänge, Kontakte.

\subsection{Sozialformen}

Früher war für den ungarischen Mathematikunterricht der Frontalunterricht typisch, also der Lehrer hielt so zu sagen einen "Vortrag". Die Persönlichkeit der Lehrer konnte sie über die Richtigkeit der Lösung überzeugen. Die Probleme des Verstehens dieses Unterrichts schärften sich in einer nicht so begabten Gruppe aus. Mit dem neuen Nationallehrplan wurde es klar, dass diese fast einzige Methode des Frontalunterrichts nicht weiter haltbar ist. Laut meiner Erfahrungen verwenden immer mehr Mathematiklehrer während des Unterrichts neue Methoden, neue Sozialformen.

Die Definition der Sozialform formuliert Becker folgenderweise: "Unter Sozialform wird die Art und Weise verstanden, in der der Lehrer die Schüler zum Lernen organisiert oder die Schüler sich selbst organisieren. Deshalb kann auch von "Organisationsformen" gesprochen werden, die durch bestimmte interaktionale Konstellationen gekennzeichnet sind." (Becker (1984) [1]) Grundsätzlich unterscheidet die Literatur heute sechs Sozialformen: Einzelarbeit (Alleinarbeit), Partnerarbeit (Paararbeit), Kleingruppenarbeit, Großgruppenunterricht (Kreisunterricht), Klassenunterricht (Frontalunterricht), Team Teaching. Während des Unterrichts habe ich außer Großgruppenunterricht und Team Teaching die anderen vier Sozialformen benutzt. Nach dem einführenden Beispiel, welches ich zum Teil an der Tafel gezeigt habe, zum Teil gemeinsam mit den Schülern "entdeckt" habe, kamen die Übungen. Bei allen Aufgaben haben die Schüler entweder in Paaren oder in 3-er Gruppen gearbeitet. Ich habe ihnen dabei freien Raum gelassen, sie konnten sich also selbst ihr(e) Partner auswählen. So wollte ich das ruhigste und somit effektivste Arbeitsklima sichern. 
2.3. Charakteristika des in der Schule unterrichteten Lehrbuches hinsichtlich des Themas

Ein Lehrbuch möchte ich ein wenig detailliert darstellen: das Lehrbuch "Sokszínü matematika" (Bunte Mathematik von Kosztolányi [2]), da in der Schule in Pilisvörösvár aus diesem Buch unterrichtet wird (sowohl in den ungarischen als auch in den bilingualen Klassen, obwohl die Sprache des Buches ungarisch ist). In diesem kurzen Überblick konzentriere ich auf den Abschnitt in diesem Buch, der in erster Linie aus der Hinsicht meiner Forschung wichtig ist, also der sich mit den exponentiellen und logarithmischen Funktionen, mit ihren Einführungen und Anwendungen beschäftigt. In den bilingualen Klassen werden der Aufbau des Lehrstoffes, die Aufgabentypen, die didaktischen Konzepte und der Stoffverteilungsplan anhand dieses Buches zusammengestellt, unterrichtet.

Dieses Lehrbuch war unter den ersten, die den Erwartungen des neuen Nationallehrplans entsprechen wollten und die damals grauen Mathematikbücher in ein bunteres umwandeln wollten. Diese schnelle Erscheinung bedeutet für das Buch sowohl einen Vor- als auch einen Nachteil. Vorteil, weil das Buch wegen der Schnelligkeit auf dem ungarischen Lehrbüchermarkt auf Anhieb beliebt wurde. Die Verfasser haben zum Teil kompetenzgeeignete Aufgaben verwendet, praktische Aufgaben gezeigt. Der Nachteil ist, dass nach diesem Buch viele neuere erschienen sind, die teilweise diese bunte, praxis- und kompetenzorientierte Sichtweise übernommen haben, teilweise haben sie sie erweitert, weiterentwickelt, viele "frische" Ideen benutzt.

Die Potenzen, Wurzelgesetze werden mit Formeln, mathematischen Mitteln wiederholt, welche von Drillaufgaben gefolgt werden. Nachher kommen die Potenzund Wurzelfunktionen, die mit "herkömmlichen" Methoden behandelt werden. Danach stehen die Potenzen mit rationalen Exponenten, die wieder mit mathematischen Formeln und Aufgaben bearbeitet werden. Mit den irrationalen Exponenten wird gleichzeitig die exponentielle Funktion eingeführt, und viele Beispielsfunktionen gezeigt. Hier kommt auch die erste praktische Anwendung, eine Aufgabe über den Luftdruck. In dem Übungsteil ist auch noch eine andere Aufgabe zu finden, über einen radioaktiven Zerfall. Leider kommen keine anderen Aufgaben solcher Art im exponentiellen Teil.

Der Logarithmus wird mit einem Textbeispiel eingeführt, Bakterien vermehren sich. Zur Lösung wird zum Teil eine Tabelle, zum Teil die exponentielle Funktion verwendet. 
Gleich danach kommen die Definition des Logarithmus und eine Reihe von Übungsaufgaben. Obwohl die Einführung der Logarithmusfunktion durch mathematische Mittel passiert, ist unter den Übungsaufgaben auch eine Textaufgabe zu finden. Unter den Logarithmusgesetzen, Gleichungen, Ungleichungen, Gleichungssystemen findet man keine praktischen Anwendungen. Nur im letzten Abschnitt sind die folgenden Typen zu sehen: Abkühlung von Materialien, Amortisation, radioaktiver Zerfall, Wachstum des Drogenkonsums, aber nur je ein Beispiel. Wie in diesem Buch zu sehen ist, sind zwar die neueren didaktischen Richtungen zu finden, bildet aber das Lehrbuch lieber einen Übergang, eine Mischung zwischen der alten, rein mathematischen Schule und den neueren, praktischen Anwendungen.

\section{Entwickelndes Unterrichtsexperiment}

\subsection{Die Rahmen des Experiments}

In meinem entwickelnden Unterrichtsexperiment versuchte ich den Lernstoff des Themenkreises Wurzel, Prozent, Exponential- und Logarithmusfunktionen in Hinsicht auf die neuesten Herausforderungen des neuen Abitur, der PISATests, aber vor allem auf die Ergebnisse der pädagogischen und psychologischen Forschungen aufzubauen.

Mein wichtigstes Ziel war dabei, dass die Schüler den Lehrstoff besser und leichter verstehen und erlernen können. Ich habe die Forschungsergebnisse, die verschiedenen mathematikdidaktischen Züge -in erster Linie die holländischen, realistisch mathematischen Methoden - verwendet, mit deren Hilfe ich den Lehrstoff, in erster Linie die Einführung neuer mathematischen Begriffe wie Exponentialfunktion, Logarithmus, Logarithmusgesetze entwickelt habe. Dabei habe ich mich stark auf die Vorteile gestützt, welche der Computer, die verschiedenen Softwares -in erster Linie GeoGebra-, die Online-Aufgaben und Übungen, die Lehrvideos bieten.

Mein entwickelndes Unterrichtsexperiment wurde im Friedrich Schiller Gymnasium in Pilisvörösvár, Ungarn, durchgeführt. Die Gruppe bestand aus 21 Schülerinnen und Schülern aus zwei verschiedenen Klassen. Die Schüler, die sich mit Mathematik beschäftigen wollten, haben den Leistungskurs gewählt, die anderen Schüler habe ich zum Teil unterrichtet. Diese Schüler waren nicht besonders gut in Mathematik, die Durchschnittsnoten am Ende des vorigen Schuljahres waren die folgenden: 
Tabelle 1. Ergebnisse am letzten Schulende

\begin{tabular}{|c|c|c|}
\hline $10 . c$ & $11 . s$ & Insgesamt \\
\hline 2,92 & 2,38 & 2,71 \\
\hline
\end{tabular}

Die Schüler beider Klassen ${ }^{2}$ haben die Mathematik ab dem 9. Jahrgang auf Deutsch gelernt, aber von verschiedenen Lehrern. In diesem neuen Jahrgang haben sie einen neuen Lehrer -mich- bekommen. Es war eine große Herausforderung die Gruppe kennen zu lernen, die Schülerinnen und Schüler miteinander, mit dem Lehrstoff und mit mir vertraut zu machen. Das Ergebnis des Vortestes ${ }^{3}$ hat gezeigt, dass es besonders große Bemühungen erfordert, um die nötigen Kenntnissen nachholen zu können.

Tabelle 2. Ergebnisse des Vortests

\begin{tabular}{|l|c|c|}
\hline $10 . c$ & $11 . s$ & Insgesamt \\
\hline 2,45 & 2,5 & 2,47 \\
\hline
\end{tabular}

Nach dem Potenzieren mit ganzen Exponenten bzw. Wurzelziehen mit "klassischen" Methoden wiederholt wurden, habe ich die exponentiellen Vorgänge schon mit realistischen Mitteln eingeführt (vgl. Várady, 2012 [7]). Die Ergebnisse haben bei dem kleineren Themenkreis (Potenzen rationalen Exponenten und n-te Wurzel) gezeigt, dass die Schüler der Gruppe in kürzeren Zeitabständen relativ gut arbeiten können.

Die Schüler hatten wöchentlich vier Mathematikstunden, für das Thema "Wurzelziehen, Potenzen mit rationalen Exponenten, Exponentialfunktionen, Logarithmusfunktionen und ihre Anwendungen" gab es laut Stoffverteilungsplan 33

${ }^{2}$ Die zwei Klassen besuchen den gleichen Lehrgang, aber die Klasse "s" hatte ein deutschsprachiges Vorbereitungsjahr, deshalb haben sie die Bezeichnung 11.s. Die Schüler sind 17, 18 Jahre alt. In Ungarn ist die beste Note 5 , die schlechteste 1.

${ }^{3}$ Durchschnitt des Tests war 46,1\%, besonders große Wissenslücken erwiesen sich auf den folgenden Gebieten:

- Potenzen mit negativen Exponenten,

- komplexere Terme mit Potenzen,

- aus dem Funktionsgraphen die Funktionsgleichung herzustellen, interessanterweise in erster Linie bei den linearen Funktionen,

- den Zusammenhang zwischen den mathematischen Sachinhalten und dem alltäglichen Leben die Zusammenhänge zu sehen (Hier haben zwar einige Schüler lineare Beispiele angegeben, sie waren aber dem Beispiel sehr ähnlich, eine andere Art linearer Zusammenhänge konnte niemand schreiben. Quadratische Beispiele habe ich überhaupt nicht gefunden.) 
Tabelle 3. Ergebnisse der Klassenarbeit Exponentielle und Logarithmusfunktionen

\begin{tabular}{|c|c|c|}
\hline $10 . c$ & $11 . s$ & Insgesamt \\
\hline 3,15 & 3,13 & 3,14 \\
\hline
\end{tabular}

Unterrichtsstunden. In der Schule wurde aus dem Lehrbuch des Mozaik Verlages unterrichtet. Der Verlag empfehlt für das Thema 25 Unterrichtsstunden für 3 Stunden pro Woche, so war die Zeit für diese Klasse 33 Stunden. Diese Zeit ist für den großen Stoff sehr knapp. Anscheinend mussten wir mehr leisten als eine "normale" Klasse, weil allein schon die Einführung nicht die herkömmliche Methode war, also ein-zwei Beispiele, dann eine Menge von Übungsaufgaben. Ich versuchte schon die Einführung so zu planen, dass durch die Aufgaben, die aus dem Alltagsleben kamen, die Schülerinnen und Schüler den Sinn des Stoffes besser verstehen, sich in ihn vertiefen, sich vertraut machen konnten.

\subsection{Der Unterrichtsplan}

Bei der Zusammenstellung des Unterrichtsplans mussten die folgenden Stichpunkte gewichtet beachtet werden:

- Der Lehrstoff muss die Erfordernisse des Abiturs erfüllen,

- Er muss flexibel sein, wo es möglich ist, muss er für das Nachholen, Einüben der fehlenden Kenntnissen Zeit und Raum bieten,

- Er darf nicht länger als der vorgeschriebene Zeitraum (33 Stunden) sein,

- Möglicherweise müssen die Bücher verwendet werden, die die Schüler schon haben,

- Die fehlenden (neuen) Materialien müssen die Schüler jede Stunde zur Hand bekommen,

- Er muss einen großen Wert -neben dem genauen mathematischen Sachverhaltauf die praktische Verwendbarkeit legen,

- Mit Hilfe der realistischen Methode und der Visualisierung, mit einer Vielfalt der Aufgaben müssen die theoretischen Kenntnisse eingeführt werden, damit zwischen den "puren" mathematischen und den alltäglichen Problemen in den Schülern feste Verbindungen entstehen können,

- Bei der Visualisierung lohnt es sich die Technik, die Computer verwenden, die Schüler dazu zu ermutigen, sie auch zu Hause, während des Lernens verwenden zu können, 
- Durch ständige Kontrolle des Lernvorgangs (Vortest, Kleintests, Klassenarbeit, Kontrollieren der Hausaufgaben) musste ich immer im Klaren sein, was die Schüler richtig, nicht so gut oder gar nichts oder falsch verstanden haben.

- Den Schülern die Angst vor Mathematik, bzw. Potenzen, exponentiellen und logarithmischen Vorgängen zu nehmen,

- Durch eine methodische Vielfalt, durch die verschiedenen Sozialformen in den Stunden die selbständige Arbeit, die kleinen Entdeckungen unterstützen zu können.

\subsection{Die Einführung des Logarithmus}

Die Schüler haben die exponentiellen Vorgänge mit Hilfe eines praktischen Beispiels kennengelernt, nämlich in einem Teich wuchsen Wasserlinsen. Wir haben dieses Beispiel sowohl graphisch, als auch algebraisch gelöst. Wir haben dazu Tabellen gefertigt, wo die ganzen (positiven aber auch negativen) Exponenten gedeutet wurden. Sogar konnten wir die rationalen Exponenten mit Hilfe dieses Beispiels einführen und darauf hinweisen, dass in diesem Beispiel auch die irrationalen Exponenten eine Bedeutung haben (Várady, F (2012) [7]). Ich wollte bei der Einführung des Logarithmus mit einem schon bekannten Problem anzufangen. Damit wollte ich mehrere Ziele erreichen:

(1) Meinen Erfahrungen nach ist der Begriff des Logarithmus für die Schüler sehr abstrakt, wenn sie keine praktischen Anwendungen sehen, verstehen sie den Begriff oft nicht (obwohl sie damit mehr oder weniger richtig rechnen können).

(2) Durch das bekannte Problem können sie sich mit Logarithmus (am Anfang noch ohne zu wissen, was das ist) vertraut zu machen.

(3) An diesem Beispiel kann man leicht und verständlich den Zusammenhang (Funktion - Umkehrfunktion) zwischen den exponentiellen und logarithmischen Rechnungen zeigen, deutlich machen.

Da die Grundaufgabe für die Schüler schon bekannt war, haben sie sich davor nicht erschrocken. Wir haben die Aufgabe noch einmal besprochen, darauf hingewiesen, dass jetzt die angegebenen Angaben und gestellten Fragen eben "umgekehrt" formuliert sind als bei den Aufgaben vorher. Deshalb sind auch die Tabellen umgekehrt aufgeschrieben.

Durch diese Methode habe ich in persönlichen Gesprächen mit den Schüler gesehen, dass der Sinn des Logarithmus für die Mehrheit der Schüler kein Problem bedeutet. Anhand dieser Einführung konnten wir in der nächsten Stunde zuerst 
Tabelle 4. Einführung der Logarithmusfunktion

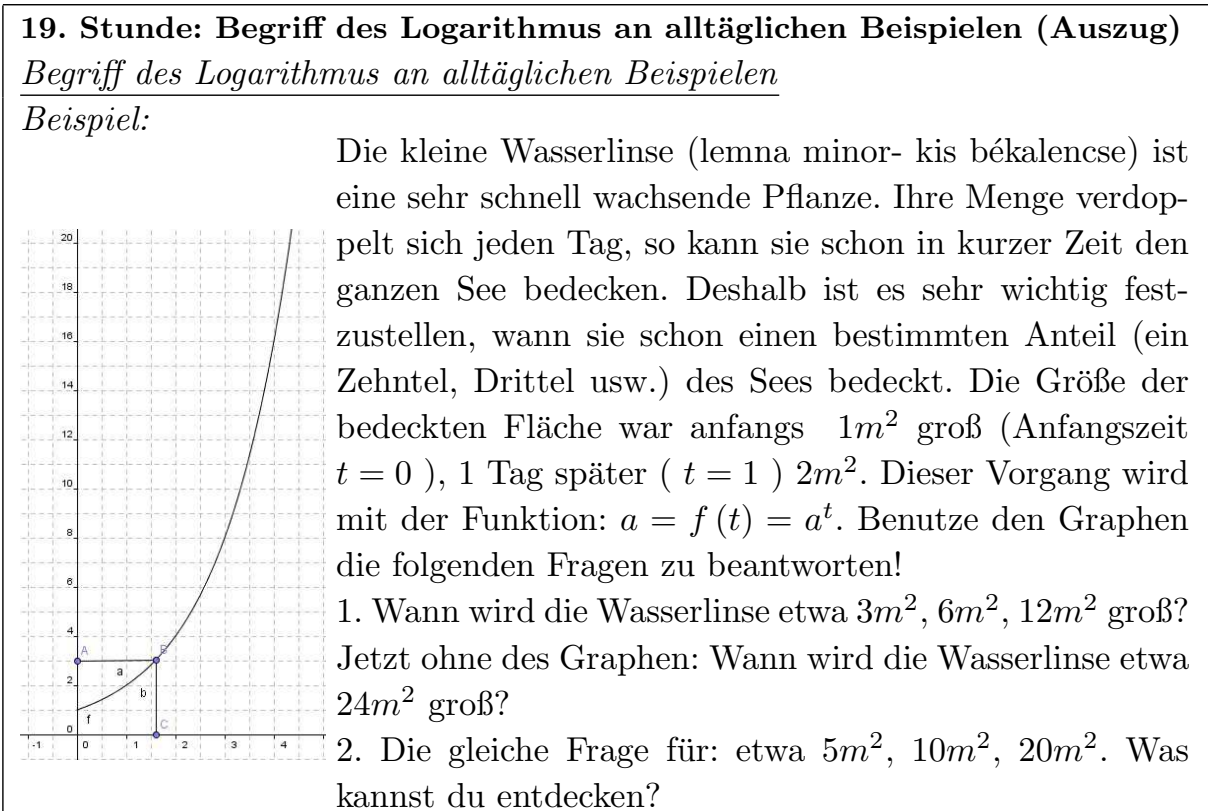

Fülle die Tabellen aus!

\begin{tabular}{|l|l|l|l|l|l|l|}
\hline Fäche $\left(m^{2}\right)$ & 1 & 2 & 4 & 8 & 16 & 32 \\
\hline Zeit (Tage) & 0 & 1 & & & & \\
\hline
\end{tabular}

\begin{tabular}{|c|c|c|c|c|c|c|}
\hline Fäche $\left(m^{2}\right)$ & 3 & 6 & 12 & 24 & 48 & 96 \\
\hline Zeit (Tage) & 1,6 & & & & & \\
\hline
\end{tabular}

Was für einem Gedankengang könntest du folgen, wenn der Graph nicht bekannt wäre?

die der Aufgabe entsprechende "Definition", dann die mathematische Definition einführen. Die Schüler haben einerseits die Definition verstanden, andererseits haben sie gesehen, wozu man sie verwenden, benutzten kann.

In der nächsten Stunde haben wir den exakten Logarithmusbegriff erlernt, dabei haben wir zuerst die schon benutzten alltäglichen Begriffe verwendet wie 


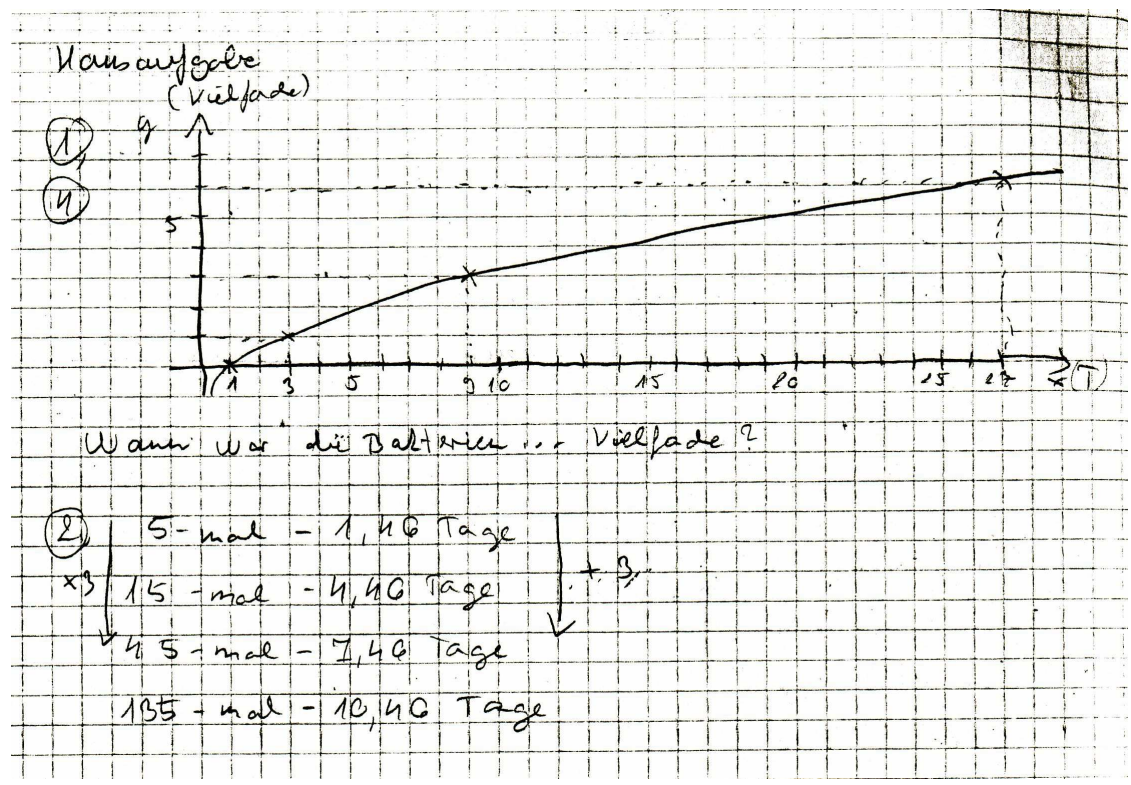

Abbildung 1. Aus den Schülerlösungen

z.B. Wachstumsfaktor, Zeitabstand. Erst dann haben wir die Definition angegeben, die meiner Umfrage in der Stunde nach die Schüler wirklich verstanden haben.

Dementsprechend wurden auch die Logarithmusgesetze besprochen. Wir sind nicht von den "reinen" mathematischen Zusammenhängen ausgegangen, sondern eben umgekehrt. Wir sind zu unserem Beispiel zurückgegangen und an diesem Beispiel wurden sie eingeführt. Natürlich, wie es auch bei der Definition des Logarithmus vorgekommen ist, wurden die Gesetze auch mathematisch beschrieben und bewiesen. Aber eben den Sinn hat die Mehrheit der Schüler gut verstanden, sie konnten sie verwenden. Das erste Gesetz wurde zum Beispiel folgendermaßen eingeführt:

Wie hier zu sehen ist, spielt beim Lernen der Text, das Textumfeld eine wesentliche Rolle. Ich habe hier Fragen gestellt, den Zusammenhang mussten die Schüler anhand des Textes verstehen und erklären. Sie haben das gerne gemacht, in Paaren versuchten sie die Antwort zu finden, und fast alle Paare haben eine richtige Antwort gegeben. Nach der Antwort mussten sie auch das -vermutete- 
Tabelle 5. Der Logarithmusbegriff

Logarithmusfunktion, Zusammenhang mit der Exponentialfunktion

Wie schon in der vorigen Stunde darauf hingewiesen wurde, wird oft bei exponentiellen Vorgängen nicht nach der Größe, sondern nach der Zeit gefragt. Man kehrt also die Richtung der Zuordnung um. Im ersten Fall spricht man über eine exponentielle Funktion, im zweiten über eine Logarithmusfunktion.

$$
\begin{array}{l|l}
t \rightarrow a \text { (Exponentialfunktion) } & a \rightarrow t \text { (Logarithmusfunktion) } \\
\hline
\end{array}
$$

In Worten:

Exponentialfunktion: alle fixen Zeitabstände bedeuten eine fixe Multiplikation der Größe.

Logarithmusfunktion: alle fixen Multiplikationen der Größe passieren in fixen Zeitabständen.

Im Beispiel der vorigen Stunde (Wasserlinsen) war der Wachstumsfaktor 2, die Bezeichnung der nötige Zeit $(t)$ für das erreichen eine bestimmet Fläche $(a)$ ist: $t=\log _{\text {Wachstumsfaktor }} a$, also in diesem Fall: $t=\log _{2} a$. Der Term z.B.: $\log _{2} 10$ bedeutet die Zeit, die die Pflanze braucht, aus der Größe von $1 m^{2}$ die Größe von $10 m^{2}$ erreichen zu können, wenn der Wachstumsfaktor 2 ist.

Definition: Unter dem Logarithmus einer positiven Zahl b zur Basis a $(a>0 ; a \neq 1)$ versteht man diejenige reelle Zahl $x$, mit der man $a$ potenzieren muss, um $b$ zu erhalten. $x:=\log _{a} b \Leftrightarrow a x=b(a, b>0 ; a \neq 1)$ (lies: " $x$ ist der Logarithmus von $b$ zur Basis $a$ genau dann, wenn $a$ hoch $x$ gleich $b$ ist.")

Gesetz formulieren. Das haben die Paare wieder richtig gemacht (alleine die Bedingungen mussten wir gemeinsam besprechen). In ähnlicher Weise sind wir auch bei den anderen Gesetzen verfahren. Die Schüler konnten die Zusammenhänge fast immer sowohl an einem konkreten Beispiel als auch im Allgemeinen richtig beantworten. 
Tabelle 6. Einführung der Logarithmusgesetze

Logarithmusgesetze:

Bei der Einführung des Logarithmus haben wir ein Beispiel über Wasserlinsen, die ihre Fläche täglich verdoppeln. Es gab eine Tabelle mit Näherungswerten dazu:

\begin{tabular}{|c|c|c|c|c|c|c|c|c|c|c|c|c|c|}
\hline $\mathrm{a}$ & 1 & 2 & 3 & 4 & 5 & 6 & 7 & 8 & 9 & 10 & 11 & 12 & 13 \\
\hline $\mathrm{t}$ & 0 & 1 & 1,6 & 2 & 2,3 & 2,6 & 2,81 & 3 & 3,17 & 3,32 & 3,46 & 3,6 & 3,7 \\
\hline $\mathrm{a}$ & 14 & 15 & 16 & 17 & 18 & 19 & 20 & 21 & 22 & 23 & 24 & 25 & 26 \\
\hline $\mathrm{t}$ & 3,81 & 3,91 & 4 & 4,1 & 4,17 & 4,25 & 4,32 & 4,39 & 4,46 & 4,52 & 4,58 & 4,6 & 4,7 \\
\hline
\end{tabular}

Weiterhin haben wir bei der Definition des Logarithmus festgestellt, dass er die Zeit $(t)$ zur Erreichen einer bestimmten Größe bedeutet, z.B.:

$\log _{2} 2=1$, d.h. wenn der Wachstumsfaktor 2 ist (Basis), dann braucht sie 1 Tag, ihre Fläche verdoppeln (2) zu können.

\section{Erkläre die folgenden Zusammenhänge:}

$$
\log _{2} 3 \approx 1,58, \log _{2} 5 \approx 2,32, \log _{2} 6 \approx 2,58
$$

Wenn wir die Tabelle weiter studieren, können wir etwas interessantes entdecken:

$$
\log _{2} 2+\log _{2} 3=\log _{2} 6 \approx 2,58
$$

Wie lässt sich das erklären?

Die Antwort ist sehr einfach: da Logarithmus eine Zeit bedeutet, zuerst braucht sie 1 Tag $\left(\log _{2} 2\right)$, ihre Fläche verdoppeln zu können, dann noch etwa 1,58 Tage $\left(\log _{2} 3\right)$, um die verdoppelte Fläche noch verdreifachen zu können. So vergingen etwa 2,58 Tage, und die Fläche ist jetzt $2 \cdot 3=6$-mal so groß.

Aus den oben erwähnten kommt das erste Gesetz des Logarithmus (L1):

(L1): $\quad \log _{a} b+\log _{a} c=\log _{a}(b ? c) \quad$ wenn $a, b, c>0$ und $a \neq 1$. 


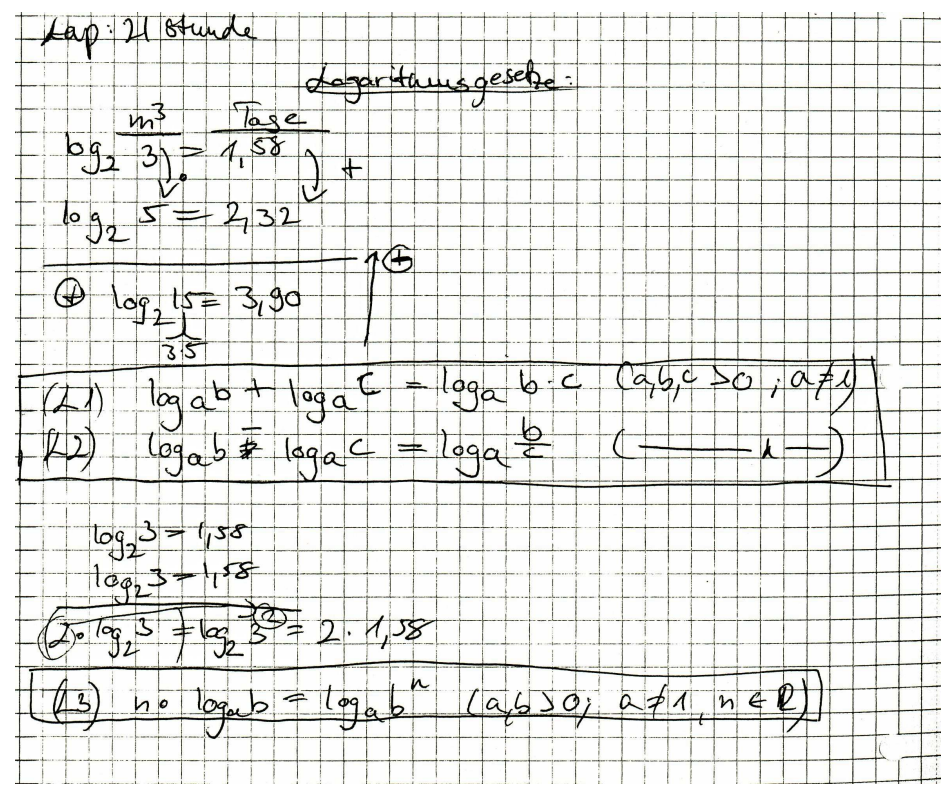

Abbildung 2. Logarithmusgesetzte

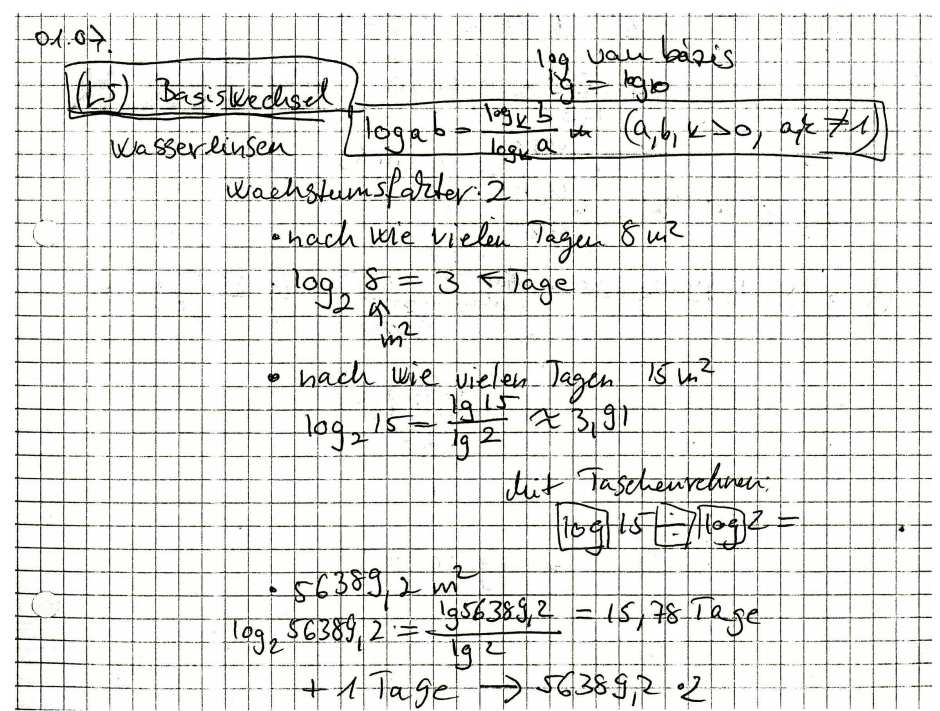

Abbildung 3. Basiswechsel 


\section{Schlussfolgerungen}

Nach langjährigen Erfahrungen habe ich immer - unabhängig von der Gruppe und von den mathematischen Fähigkeiten der Schüler - die Erfahrung gemacht, dass das Thema Logarithmus für die Mehrheit der Schüler zu abstrakt, zu unverständlich und zu überflüssig war und blieb. Mit der Einführung des neuen Abiturs, als auch auf diesem Gebiet praktische Aufgaben eingeführt wurden, haben die Schüler die Nützlichkeit dieses Teilgebietes der Mathematik besser verstanden. Wegen der Schwierigkeiten der abstrakten Bezeichnungen und der ungewöhnlichen Rechnungsarten des Logarithmus (s. die Logarithmusgesetze) blieb das Thema für die meisten Schüler eher fremd. Deshalb habe ich eben das als didaktisches Forschungsgebiet gewählt. Durch eine Mischung des ungarischen Unterrichtsplans und der ungarischen Methoden mit den Zügen der Freudental'schen Schule versuchte ich den Logarithmus den Schüler näher zu bringen, verständlicher zu machen. Aus den persönlichen Gesprächen mit den Schülern, aus den Erfahrungen in den Stunden, aus den Ergebnissen der Tests wurde mir klar, dass es die Mühe lohnt, die reine Theorie mit praktischen Beispielen zu bearbeiten, die Zusammenhänge mit Textumfeld einzuführen, die Möglichkeit den Schülern anzubieten die Gesetze entdecken und formulieren zu können. In diesem schweren und abstrakten Thema war der Durchschnitt des Tests der Gruppe 3,14, was um 0,43 (um fast 16\%) besser war als die Ergebnisse des vorigen Jahres. Wenn man dieses Ergebnis sogar mit dem Ergebnis des Vortest vergleicht, kann man die Entwicklung noch gravierender sehen: der Durchschnitt wuchs um 0,53, was einem prozentualen Wachstum von $27 \%$ bedeutet. Im Nachtest, in der letzten Aufgabe, wo ein alltägliches Beispiel aufgegeben wurde, haben sie $87 \%$ der Schüler den ersten Teil angefangen und fast alle haben ihn richtig gelöst. Die Frage b) wurde von $47 \%$ der Schüler gelöst, und der dritte Teil, wo Logarithmus verwendet werden soll, haben $33 \%$ der Schüler richtig gelöst. Ich möchte aber betonen, dass der erstrangige Vorteil dieser Methode für mich nicht unbedingt das bessere Ergebnis des Testes war, was natürlich auch sehr wichtig ist, sondern, dass die Schüler selbst Fragen stellen können, Gesetze entdecken können und sie formulieren können. Dadurch wird ihre Angst gegenüber der Mathematik abgebaut, sogar können sie auch die Schönheiten des Denkens entdecken. 
Tabelle 7. Letzte Frage des Nachtests

Herr Zweifel überlegt, was er mit 30000 anfangen soll.

a) Bei welchem Zinssatz würde er in 6 Jahren $40 \%$ mehr Geld besitzen?

b) Welchen Betrag müsste er heute bei einem Zinssatz von 4,5\% anlegen, um in 15 Jahren 30000 ausgezahlt zu bekommen?

c) In wie vielen Jahren wächst das Geld bei einem Zinssatz von $5 \%$ mindestens auf das Dreifache des ursprünglichen Wertes?

\section{Literatur}

[1] G. E. Becker, Planung von Unterricht, Handlungsorientierte Didaktik, Teil I, 104, Beltz Verlag, Weinheim und Basel, 1984.

[2] J. Kosztolányi, I. Kovács, K. Pintér, J. Urbán and I. Vincze, Sokszinü matematika (középiskola 11. osztály), MOZAIK Kiadó, Szeged, 2003.

[3] J. de Lange, Using and Applying Mathematics in Education, in: International handbook of mathematics education, Part one, (A.J. Bishop, et al., eds.), Kluwer Academic Publisher, 1996, 49-97.

[4] G. Roth, Neurobiologische und psychologische Grundlagen des Lehrens und Lernens am Beispiel der Mathematik, (2012.11.12), 16, http://www.klett-mint.de /fileadmin/Kongresse/MINT_Kongress_2010/5_Prof_Roth_Lehren_und_Lernen.pdf.

[5] R. R. Skemp, A matematikatanulás pszichológiája, Edge 2000 Kiadó, Budapest, 2005.

[6] A. Treffers, Three dimensions: a model of goal and theory description in mathematics instruction - The Wiskobas project, Kluwer Academic Publishers, Dordrecht, 1987.

[7] F. Várady, Teaching exponential and logarithmic functions and their applications through the software GeoGebra, ISBN 978-963-661-988-6, University of Miskolc, 2012 .

FERENC VÁRADY

BUDAPEST BUSINESS SCHOOL

COLLEGE OF COMMERCE CATERING AND TOURISM

1054 BUDAPEST, ALKOTMÁNY UTCA 9-11.

HUNGARY

E-mail: varady.ferenc@kvifk.bgf.hu

(Received January, 2013) 